Service social

\title{
Portrait des programmes québécois d'aide aux conjoints ayant des comportements violents
}

\author{
Normand Brodeur, Élisabeth Lesieux, Maryse Rinfret-Raynor et Joannie \\ Pépin-Gagné
}

Volume 60, numéro 1, 2014

Des enfants, des couples et des familles.

URI : https://id.erudit.org/iderudit/1025130ar

DOI : https://doi.org/10.7202/1025130ar

Aller au sommaire du numéro

Éditeur(s)

École de service social de l’Université Laval

ISSN

1708-1734 (numérique)

Découvrir la revue

Citer cet article

Brodeur, N., Lesieux, É., Rinfret-Raynor, M. \& Pépin-Gagné, J. (2014). Portrait des programmes québécois d'aide aux conjoints ayant des comportements violents. Service social, 60(1), 1-14. https://doi.org/10.7202/1025130ar

\section{Résumé de l'article}

Trente-trois organismes communautaires québécois ont un programme d'aide aux conjoints ayant des comportements violents envers leur partenaire. L'objectif de l'étude présentée dans cet article était de dresser un portrait de leurs services et d'examiner les effets du financement qui leur a été octroyé par le gouvernement du Québec dans le cadre de son deuxième plan d'action en matière de violence conjugale (2004-2009). Vingt-sept organismes ont complété un sondage réalisé à la fin de l'année 2008. Les résultats sont analysés à la lumière d'études similaires réalisées aux États-Unis et en Europe. 


\title{
Portrait des programmes québécois d'aide aux conjoints ayant des comportements violents
}

\author{
BRODEUR, Normand \\ LESIEUX, Élisabeth \\ RINFRET-RAYNOR, Maryse \\ PÉPIN-GAGNÉ, Joannie
}

\section{RÉSUMÉ}

Trente-trois organismes communautaires québécois ont un programme d'aide aux conjoints ayant des comportements violents envers leur partenaire. L'objectif de l'étude présentée dans cet article était de dresser un portrait de leurs services et d'examiner les effets du financement qui leur a été octroyé par le gouvernement du Québec dans le cadre de son deuxième plan d'action en matière de violence conjugale (2004-2009). Vingt-sept organismes ont complété un sondage réalisé à la fin de l'année 2008. Les résultats sont analysés à la lumière d'études similaires réalisées aux États-Unis et en Europe.

Mots-clés : Violence conjugale - Québec - programmes pour hommes

\begin{abstract}
In Quebec, thirty-three community organizations operate an intervention program for men who use violence in their intimate relationships. The aims of the present study were to describe the services they offer and to examine the effects of the financial support they were given by the Government of Québec in its second action plan on domestic violence (2004-2009). Twentyseven organizations were surveyed at the end of 2008. The results are discussed in the context of similar studies conducted in the United States and in Europe.
\end{abstract}

Keywords: Intimate partner violence - Quebec - men's programs 
Les programmes d'aide aux conjoints ayant des comportements violents ont été créés aux États-Unis à la fin des années 1970 afin de contrer la violence conjugale. Ils sont ensuite apparus dans d'autres pays, dont le Canada. À la suite de l'adoption de politiques d'arrestation obligatoire et de l'imposition d'ordonnances de traitement par les tribunaux, le nombre d'hommes qui y sont adressés s'est accru substantiellement à partir des années 1980, ce qui a entraîné la multiplication des programmes (Price et Rosenbaum, 2009). Il y en a 2000 en sol américain (Dalton, 2007), plus de 190 dans les pays de l'Union européenne (WWP, 2008a) et 205 au Canada, dont une trentaine dans la province de Québec (Gouvernement du Canada, 2008).

Les programmes visent l'arrêt des conduites abusives en intervenant sur la résolution des conflits, la gestion des émotions et de la colère, la responsabilisation des individus face à leurs gestes violents et l'acquisition de compétences sociales (Mathieu, Bélanger et Brisebois, 2006). Tout en ayant une mission commune, les programmes sont diversifiés (Rosenbaum et Leisring, 2002). Chacun s'adapte aux conditions particulières de son environnement telles que les ressources disponibles, les politiques sociales et pénales, ainsi que les attentes de la communauté dans laquelle ils interviennent (Gondolf, 2002 : 156). Il existe toutefois relativement peu d'études sur la façon dont les services sont organisés dans différents contextes sociaux. Cette rareté est particulièrement flagrante au Canada où, à notre connaissance, aucune étude récente ne présente un portrait des programmes offerts sur les plans national ou provincial. Cet article entend combler partiellement ce vide en présentant les résultats d'une étude portant sur les programmes québécois et sur l'influence du financement qui leur a été octroyé dans le cadre du deuxième plan d'action gouvernemental en matière de violence conjugale (Gouvernement du Québec, 2004).

\section{Les programmes d'aide aux conjoints ayant des comportements violents}

Les recherches qui dressent le profil des programmes d'aide aux conjoints ayant des comportements violents sont peu nombreuses. Aux États-Unis, trois sondages nationaux décrivant les programmes ont été réalisés au début des années 1980 ; il s'est ensuite écoulé plus de vingt ans avant que Dalton (2007) publie une étude similaire. Dans cette enquête auprès de 150 programmes, l'auteur examine plus spécifiquement la structure des programmes, les services offerts, les processus d'aiguillage des clients vers d'autres services, les caractéristiques des participants, l'évaluation des activités et la récidive. Une autre enquête a été menée par Price et Rosenbaum (2009) auprès de 276 programmes provenant de 45 États américains. Ces auteurs ont étudié les grandes orientations des programmes, leur structure, le leadership, les activités et le financement. Dans les pays de l'Union européenne, un sondage a été mené en 2008 auprès de 192 programmes disséminés dans 19 pays (WWP, 2008a). Les questions portaient sur les pratiques, les approches et les conditions dans lesquelles ils évoluent.

Selon ces études, le groupe est la modalité d'intervention la plus fréquente. Aux États-Unis, plus de $95 \%$ des hommes sont traités de cette façon (Price et Rosenbaum, 2009). Bien que des interventions individuelles ou de couple soient offertes en complément dans certains programmes, elles occupent une place beaucoup moins grande dans l'offre de service. La durée des programmes basés sur l'intervention de groupe varie par ailleurs grandement. Dalton (2007) 
en situe l'étendue entre 8 et 52 semaines, la moyenne étant de 31,5. Les tendances observées en Europe sont semblables. Les interventions de groupe y sont toutefois plus courtes, la majorité d'entre elles ayant entre 14 et 26 semaines (WWP, 2008a).

Au niveau européen, la thérapie cognitive-comportementale (TCC) est l'approche la plus utilisée dans les programmes. En fait, près de la moitié (45\%) d'entre eux ont recours à cette forme d'intervention (WWP, 2008a). Du côté américain, le terme psychoéducatif est le descriptif le plus souvent mentionné (59\%) par les répondants du sondage de Price et Rosenbaum (2009), suivi de près par le modèle de Duluth (53\%) et la thérapie cognitive-comportementale (49\%). Le modèle de Duluth est basé sur une approche de conscientisation dans laquelle l'intervenant confronte les croyances des hommes concernant le pouvoir, le contrôle et la domination envers leurs épouses (Barner et Carney, 2011). Bien que les conjoints ayant des comportements violents soient un groupe hétérogène (Holtzworth-Munroe et Meehan, 2004), la majorité des programmes américains offrent un seul et même traitement pour tous ; selon Price et Rosenbaum, seulement $10 \%$ d'entre eux adaptent l'intervention aux besoins ou aux caractéristiques de la clientèle. Cette proportion est légèrement plus élevée dans l'étude de Dalton (2007) dans laquelle 39 des 150 répondants ont indiqué varier la longueur du traitement ou l'approche théorique utilisée selon la gravité des gestes de violence, la récidive ou la concomitance d'autres problèmes.

Tant en Europe qu'aux États-Unis, la clientèle des programmes est composée majoritairement d'hommes envoyés par le système judiciaire à la suite d'agressions envers leur conjointe (WWP, 2008a ; Price et Rosenbaum, 2009). Dans le cas américain, presque la totalité des conjoints (96\%) y arrivent dans ces conditions. Bien qu'ils s'adressent principalement à des hommes ayant agressé leur partenaire dans le cadre d'une relation hétérosexuelle, la majorité des programmes ou des organismes qui les hébergent offrent également des services à d'autres populations. En effet, en Europe, $44 \%$ des organismes offrent aussi un service aux femmes auteures de violence conjugale, $47 \%$ offrent un soutien aux femmes victimes de violence conjugale et $33 \%$ offrent un service aux hommes victimes de violence conjugale (WWP, 2008a). Aux États-Unis, ces proportions sont respectivement de $73 \%, 43 \%$ et $28 \%$ (Dalton, 2007).

La plupart des programmes engagent du personnel qualifié ayant effectué des études universitaires. Plus des deux tiers des organismes américains (71\%) comptent parmi leur personnel au moins un employé ayant un diplôme de maîtrise, tandis que $27 \%$ ont au moins un employé détenant un doctorat (Price et Rosenbaum, 2009). Le personnel comprend des intervenants des deux sexes, puisque la coanimation mixte constitue la forme d'animation des groupes la plus souvent rapportée tant aux États-Unis qu'en Europe (WWP, 2008a). On ne connaît cependant pas exactement la proportion d'hommes et de femmes qu'on y retrouve.

Aux États-Unis, le financement des programmes provient principalement des honoraires versés par les participants. Ceux-ci représentent en moyenne $74 \%$ des revenus (Dalton, 2007) et la seule source dans plus de la moitié des cas (54\%; Price et Rosenbaum, 2009). En Europe, moins de $40 \%$ des programmes recueillent des honoraires des participants. L'État contribue davantage, $33 \%$ des programmes étant financés par des fonds publics provenant des 
gouvernements nationaux, régionaux et municipaux. Par contre, seulement $23 \%$ bénéficient d'un financement stable (WWP, 2008a).

\section{AU QUÉBEC}

Les programmes d'aide aux conjoints ayant des comportements violents sont apparus au Québec en 1982. Une première enquête concernant 16 programmes a été publiée en 1989 afin de décrire l'évolution rapide du secteur (Rondeau, 1989). Elle montrait qu'une partie des programmes $(n=10)$ avaient été créés dans le secteur communautaire, alors que les autres $(n=6)$ avaient été développés dans des établissements du réseau de la santé et des services sociaux. L'auteur soulignait l'absence de planification et de moyens financiers adéquats dans le développement des programmes et invitait les autorités politiques à s'impliquer davantage dans le dossier. Trois ans plus tard, le ministère de la Santé et des Services sociaux adoptait un document d'orientation reconnaissant leur rôle « essentiel et complémentaire » par rapport aux interventions effectuées auprès des femmes victimes de violence conjugale (MSSS, 1992 : 17). Ce rôle a été confirmé en 1995 par l'adoption d'une Politique d'intervention en matière de violence conjugale (Gouvernement du Québec, 1995). La reconnaissance politique des programmes a été suivie d'un engagement financier envers les organismes communautaires qui dispensaient les programmes et a permis la création de nouveaux organismes dans les régions où il n'en existait pas. En 2008, 33 organismes étaient en opération.

À la suite de l'adoption de sa politique de 1995, le gouvernement québécois a mis en œuvre des plans d'action visant à consolider la notion de responsabilité sociale et collective quant à l'élimination de la violence conjugale. Alors que le premier plan couvrait la période de 1996 à 2001, le second plan portait sur la période 2004-2009 (Gouvernement du Québec, 2004). La mesure 19 de ce second plan consistait à offrir un soutien financier accru aux organismes offrant des services psychosociaux en violence conjugale afin qu'ils « répondent aux besoins d'accueil, de soutien, de référence, d'accompagnement et de suivi » (p. 10) de toutes les personnes concernées. Au cours de cette période de cinq ans, le gouvernement a haussé de 30 millions de dollars le financement des maisons d'hébergement et de 1,5 million celui des organismes d'aide aux conjoints ayant des comportements violents. En prévision du dépôt d'un troisième plan d'action (Gouvernement du Québec, 2012), le Secrétariat à la condition féminine et le ministère de la Santé et des Services sociaux ont commandé un portrait des services offerts aux auteurs et aux victimes de violence conjugale et un bilan de l'impact du financement supplémentaire octroyé dans le deuxième plan d'action. Les résultats détaillés de cette étude ont été présentés ailleurs (Rinfret-Raynor, Brodeur, Lesieux et Turcotte, 2010). Cet article synthétise les données relatives aux programmes d'aide aux conjoints ayant des comportements violents.

\section{MÉTHODOLOGIE}

Le sondage a été réalisé à la fin de l'année 2008 auprès des organismes offrant des services d'aide aux conjoints aux comportements violents. Il visait à recueillir des données rétrospectives sur deux exercices financiers distincts, soit 2002-2003 (Temps 1 - T1), qui précédait le plan d'action gouvernemental, et l'exercice 2007-2008 (Temps 2 - T2) où toutes les sommes additionnelles promises par le gouvernement avaient été octroyées. Cette stratégie permettait 
d'établir des comparaisons entre les activités offertes avant et après le plan d'action gouvernemental et de faire ressortir l'évolution des programmes au cours de la période visée.

La majorité des données recueillies dans le sondage étaient quantitatives. Le questionnaire comprenait des questions fermées sur : les services offerts ; la disponibilité et l'accessibilité de ces services; les activités de promotion, de prévention et de collaboration réalisées dans la communauté; la situation financière des organismes; et les ressources humaines. Des données qualitatives ont aussi été recueillies afin de connaître la perception des répondants quant aux effets du financement supplémentaire sur chacun des principaux thèmes.

Le questionnaire a été envoyé aux 33 organismes du Québec. Vingt-sept organismes provenant de 15 des 17 régions administratives de la province l'ont complété. Il s'agit d'un taux de réponse élevé $(81,8 \%)$ en comparaison avec ceux obtenus dans les sondages américains évoqués plus haut (20\% pour Price et Rosenbaum, 2009 ; $49 \%$ pour Dalton, 2007). Les personnes qui ont complété le questionnaire au nom de leur organisme étaient des hommes (74,4\% des cas) occupant un poste de direction $(59,2 \%)$ ou de coordination $(29,6 \%)$. La majorité des organismes $(59,2 \%)$ ayant participé à l'étude avaient plus de quinze ans d'existence ; seulement 14,8\% d'entre eux en avaient moins de dix.

Lors de la construction du questionnaire, l'association «à cœur d'homme», qui regroupe les organismes d'aide aux conjoints ayant des comportements violents, a été consultée à propos du type de données rétrospectives qui pouvaient être récoltées. Malgré ces précautions, certains organismes ne disposaient pas des informations demandées à cause de lacunes dans leur système d'archivage ou du manque d'uniformité entre les données colligées par les différents organismes. Compte tenu de ces données manquantes, certains des résultats présentés dans cet article sont basés sur des données partielles.

\section{RÉSULTATS}

\section{Situation en 2007-2008}

\section{Populations desservies par les organismes}

Selon les données du sondage, la majorité $(n=15)$ des organismes traite entre 100 et 250 demandes d'aide par année ; neuf en traitent plus de 250, alors que trois en traitent moins de 100. Les estimations réalisées à partir de données partielles $(n=21)$ permettent de constater que $60 \%$ de ces demandes proviennent d'hommes ayant des comportements violents envers leur partenaire. Les autres demandes proviennent de groupes ayant des problèmes connexes pour lesquels les organismes offrent des services distincts. Les hommes en difficulté constituent le plus important de ces groupes (31\% des demandes). On entend notamment par hommes en difficulté ceux qui sont aux prises avec une rupture ou d'autres difficultés conjugales, sans qu'il y ait présence de violence. Les adolescents ayant des comportements violents $(5,8 \%$ des demandes), les femmes ayant des comportements violents (2,5\% des demandes) et les hommes victimes de violence conjugale $(0,7 \%$ des demandes) constituent les autres groupes desservis. 
Interventions auprès des conjoints ayant des comportements violents

Les organismes offrent aux conjoints huit types d'activités. Outre les entrevues d'accueil et d'évaluation, qui sont communes à tous, les plus courantes sont l'orientation des personnes vers d'autres ressources, le suivi individuel et les groupes de thérapie que l'on retrouve dans la quasi-totalité des cas $(92,6 \%)$. L'intervention téléphonique $(85,2 \%)$, les groupes de deuxième étape pour ceux qui ont complété le programme de base $(40,7 \%)$, les groupes de pré-thérapie $(18,5 \%)$ et la thérapie familiale ou de couple $(11,1 \%)$ sont offerts dans des proportions moindres. La gamme de services de chaque organisme comprend typiquement entre 5 et 7 de ces activités.

Selon les réponses fournies par 15 organismes, $42,3 \%$ des personnes qui participent aux programmes d'aide aux conjoints y sont dirigés par les établissements du réseau de la santé et des services sociaux alors que $34,3 \%$ le sont par le système judiciaire. Le programme typique est d'une durée de 20 rencontres de groupe, ceux qui souhaitent approfondir leur réflexion et consolider leurs acquis pouvant toutefois prendre part à des séances supplémentaires.

Tous les organismes sondés dispensent des services en français, alors qu'un peu plus du quart d'entre eux (25,9\%) offrent aussi des services en anglais. Un seul organisme propose des groupes en espagnol. Bien que trois autres se disent en mesure de rencontrer individuellement des hommes parlant d'autres langues en ayant recours à des interprètes, on constate que l'accès des néo-Québécois qui ne maîtrisent pas les langues officielles est limité. Un autre volet de l'accessibilité aux services concerne la capacité d'accueillir les conjoints aux comportements violents lors de situations de crise. Près de la moitié (44\%) des organismes ont des ententes de service avec le centre de crise de leur région ou ont mis en place d'autres mesures visant à répondre aux situations urgentes. Enfin, toujours en lien avec l'accessibilité, on observe des variations dans les délais requis pour obtenir une entrevue initiale et pour intégrer un groupe. Bien que plus de la moitié $(53,8 \%)$ des organismes soient en mesure de proposer une entrevue d'accueil dans la semaine suivant la réception d'une demande d'aide, 15,4 \% d'entre eux rapportent un délai supérieur à deux semaines. Par ailleurs, une fois le processus d'évaluation terminé, l'attente médiane pour intégrer un groupe est onze jours. Un quart des organismes déclare toutefois des délais supérieurs à trente jours.

\section{Prévention, sensibilisation et concertation}

En plus des services directs aux conjoints ayant des comportements violents, les organismes réalisent plusieurs activités de prévention de la violence dans la communauté. L'école est le milieu le plus souvent ciblé : un peu plus de la moitié des organismes (51,8\%) y intervient pour éduquer à la non-violence, soutenir les adolescents exposés à la violence conjugale ou traiter des jeunes aux comportements violents. Des activités de sensibilisation à la violence conjugale sont également effectuées auprès d'autres organismes communautaires, des établissements de santé et de service sociaux, du système correctionnel, des centres jeunesse et du grand public. La concertation avec d'autres organismes complète ce travail communautaire, chaque organisme participant annuellement à un nombre médian de sept rencontres de ce type dans son milieu. 


\section{Ressources humaines}

Les organismes emploient en moyenne 7,26 personnes. Les salariés sont principalement des hommes (64\%), qui détiennent un diplôme d'études universitaires (75,3\%) ou collégiales $(18,5 \%)$. Un peu plus de la moitié d'entre eux $(54,2 \%)$ travaille à temps partiel pour un salaire horaire qui varie entre $19,04 \$$ et 22,79 (en dollars de 2008). Environ le tiers $(36,7 \%)$ des employés travaillent au sein de leur organisme depuis plus de quatre ans tandis que 41,9\% d'entre eux le font depuis moins de deux ans. La majorité $(59,9 \%)$ des organismes ont un régime d'assurance collective pour leurs employés, mais seulement un quart $(27,3 \%)$ a un régime de retraite.

\section{Sources de financement}

Les subventions publiques constituent la principale source de revenu $(74,5 \%)$ des organismes sondés. Les sources privées (12,7\%), l'autofinancement $(6,6 \%)$ et les honoraires versés par les participants (6,2\%) complètent le financement. Au cours de l'exercice 2007-2008, le budget moyen des organismes était de 244667 \$. L'écart-type de 176125 \$ reflète des variations importantes entre les organismes.

\section{Évolution de la situation entre le Temps 1 et le Temps 2}

Sous l'effet du plan d'action gouvernemental en matière de violence conjugale, le financement public des organismes a augmenté entre le T1 et le T2. Concrètement, la subvention gouvernementale médiane s'est accrue de 36471 \$ par organisme. Pour plus de la moitié des organismes, cela représentait une hausse de moins de $30 \%$ du financement public étalée sur cinq ans. Le financement supplémentaire ayant été accordé sur la base d'un indice populationnel, les organismes situés dans les centres urbains ont toutefois reçu des montants plus substantiels que ceux situés dans les zones rurales. Leur financement public s'est accru de près de $67 \%$.

\section{Services aux conjoints}

Au cours de la période étudiée, près de $80 \%$ des organismes ont connu une hausse des demandes d'aide dont le nombre médian est passé de 126 à 175 par année, une augmentation de $39 \%$. Malgré le volume de demandes accru, plusieurs organismes ont aussi ajouté des activités à leur gamme de services. Par exemple, six organismes supplémentaires ont implanté des groupes de deuxième étape et quatre autres ont commencé à proposer des entrevues de suivi individuel à leurs clients. Ces entrevues n'ont d'ailleurs pas seulement été offertes par un plus grand nombre d'organismes. Elles ont aussi été plus fréquentes. Selon les données fournies par 24 répondants, le nombre médian est passé de 40,5 à 156,5 par année, alors que le nombre médian de participants en ayant bénéficié est passé de 13 à 35 par organisme.

Au cours de la période étudiée, l'accessibilité aux services s'est légèrement améliorée. Ainsi, le nombre d'organismes offrant des services en langue anglaise est passé de 3 au T1 (11,5\%) à 7 au T2 (26,9\%). Le nombre médian d'heures de disponibilité des intervenants pour faire des entrevues face à face lors de situations de crise est passé de 16 à 30 heures par semaine. Finalement, des données partielles ( $n=16$ organismes) indiquent que le délai d'attente médian 
entre la fin du processus d'accueil des participants et leur intégration à un groupe a été réduit de 14,5 (T1) à 10 jours (T2).

\section{Autres activités}

Entre les deux périodes étudiées, le nombre d'organismes impliqués dans des activités de prévention et de sensibilisation auprès du grand public, du milieu scolaire, des organismes communautaires, du système correctionnel, de l'appareil judiciaire, de la police, des centres jeunesse et d'autres milieux a connu de légères hausses ou est demeuré stable. Le nombre total d'activités réalisées a toutefois diminué de $15 \%$ (567 au T2 contre 670 au T1). La concertation avec d'autres organismes a, quant à elle, été plus fréquente, le nombre médian de rencontres auxquelles les organismes ont participé étant passé de quatre au T1 à sept au T2.

\section{Ressources humaines et conditions de travail}

La hausse du financement public a eu des impacts sur les ressources humaines des organismes. Les statistiques compilées pour 25 organismes indiquent que le nombre médian de postes est passé de quatre et demi au T1 à sept au T2. Pour l'ensemble de ces organismes, le nombre de postes à temps plein est passé de 59 à 77, alors que le nombre de postes à temps partiel est passé de 77 à $100^{1}$. Le profil des salariés n'a cependant pas connu de changement radical au cours de la période de référence.

Les conditions de travail des employés se sont améliorées entre le T1 et le T2. D'une part, les taux horaires ont augmenté pour la plupart des catégories de postes. Le salaire moyen des directeurs est passé de 21 \$ à 25 \$ l'heure, tandis que celui des intervenants est passé de 16 \$ à 18 \$. On observe d'autre part un investissement accru des organismes dans la formation donnée aux intervenants. En effet, les sommes consacrées à ce poste budgétaire ont augmenté d'au moins $10 \%$ dans la majorité des organismes (77,3\%); pour un peu plus du tiers de l'échantillon (36,4\%), la hausse était supérieure à $100 \%$. Le montant médian consacré aux formations s'élevait à 2500 \$ au T2. Les principales modalités étaient la supervision, la participation à des colloques et séminaires et la formation sur les lieux de travail.

\section{Opinion des répondants sur les effets du plan d'action gouvernemental}

En réponse aux questions ouvertes concernant les effets du plan d'action gouvernemental qui a permis de hausser le financement de leur organisme, les répondants ont souligné des gains qui sont de l'ordre de la consolidation des organismes et des services qu'ils offrent. Ainsi, les montants reçus ont permis à certains organismes de réduire un déficit, d'atteindre un équilibre budgétaire, voire d'éviter une fermeture. D'autres disposent maintenant de locaux ou de matériel mieux adaptés à leurs besoins. La stabilisation des équipes de travail, la bonification des conditions salariales, l'amélioration de l'encadrement clinique, la mise en place de nouvelles relations partenariales et la concertation avec d'autres organismes ont également été présentés comme des changements positifs. Sur le plan des services eux-mêmes, les répondants ont

\footnotetext{
1 Pour une comparaison historique, notons que Rondeau avait répertorié 10 postes à temps plein et 38 postes à
} demi-temps ou à temps partiel dans les 16 organismes qu'il a sondés en 1989. 
mentionné la plus grande disponibilité de leurs activités, notamment par la prolongation des heures de service, l'augmentation de la taille des groupes et du nombre d'entrevues individuelles qui sont faites auprès des conjoints ayant des comportements violents.

En dépit du financement additionnel qui leur a été accordé, les répondants ont exprimé diverses préoccupations. Certains soulignent que leur organisme demeure dépendant de subventions ponctuelles pour boucler son budget. D'autres estiment que les salaires sont toujours trop faibles en comparaison avec ceux versés aux professionnels qui travaillent dans le réseau institutionnel des services sociaux. Dans ce contexte, la capacité de recruter et de retenir du personnel qualifié demeure un sujet d'inquiétude. Les répondants indiquent aussi qu'il est difficile de répondre à toutes les demandes d'aide qui sont adressées à leur organisme et à tous les besoins. À titre d'exemple, certains ont mentionné la difficulté de desservir leur vaste territoire ou encore de répondre efficacement aux demandes des hommes vivant des réalités particulières tels que les hommes immigrants et ceux qui sont eux-mêmes victimes de violence conjugale.

Les réponses aux questions témoignent par ailleurs des effets inégaux du plan d'action sur les organismes. Bien qu'une majorité reconnaisse certaines avancées, des répondants provenant de petits organismes situés en milieu rural ont déclaré que le plan d'action n'avait eu aucun effet positif pour eux; cela reflète les écarts importants dans les montants attribués à chacun en vertu de la répartition des fonds selon un indice populationnel. Les disparités entre les organismes ont aussi donné lieu à d'autres contradictions dans la perception des effets du plan d'action. Si certains pensent que le financement a permis aux intervenants de leur organisme d'être plus disponibles pour faire des activités de prévention et de sensibilisation à la violence dans leur communauté, d'autres estiment au contraire qu'ils manquent de fonds pour ce faire, toutes leurs ressources devant être utilisées pour répondre aux demandes d'aide directes.

\section{DISCUSSION}

À tous les deux ans, Statistique Canada recueille des données sur les maisons d'hébergement pour femmes violentées opérant en territoire canadien. Son enquête aide « les fournisseurs de services, les organismes sans but lucratif et les gouvernements à mettre sur pied des programmes, élaborer des politiques et organiser des services à l'intention des femmes victimes de violence et de leurs enfants » (Statistique Canada, 2011). II n'existe pas de mesure semblable permettant de suivre l'évolution des programmes d'aide aux conjoints ayant des comportements violents. L'étude rapportée dans cet article répond en partie à ce besoin de suivi en étant la première depuis 1989 à établir un portrait des organismes qui dispensent de tels programmes au Québec. Elle revêt un caractère original du fait qu'elle visait à mesurer l'impact du financement accordé aux organismes dans le cadre d'un plan d'action gouvernemental.

L'une des limites de cette étude est liée aux lacunes constatées dans les systèmes de collecte et d'archivage des données dans les organismes visés par l'enquête. Ces lacunes ont empêché les auteurs d'avoir des données exhaustives pour toutes les variables mesurées, ce qui réduit la portée des résultats. Elles sont apparues particulièrement évidentes par rapport aux 
données qui visaient l'année 2002-2003. Comme le stipulent les normes européennes, la documentation des services rendus aux conjoints aux comportements violents est une partie intégrante de l'intervention auprès d'eux et doit être planifiée (WWP, 2008b). Ce travail de collecte systématique des données peut s'avérer plus difficile dans les petits organismes communautaires dont les ressources humaines sont restreintes. Un soutien logistique et financier peut s'avérer nécessaire pour les aider à bien l'accomplir dans l'avenir.

Une autre limite a trait au contenu de l'étude. Celle-ci s'intéressait davantage aux activités produites par les organismes qu'aux dimensions philosophiques de leur intervention. Ainsi, contrairement à d'autres enquêtes similaires, aucune donnée n'a été recueillie à propos des approches de traitement privilégiées. Une étude réalisée au milieu des années 1990 indiquait la diversité de celles-ci (Rondeau, Lindsay, Beaudoin et Brodeur, 1997). Les approches cognitivecomportementale, psychoéducative et proféministe (de type Duluth), auxquelles la documentation étatsunienne réfère abondamment, y étaient mentionnées par les répondants. Les approches humaniste et systémique l'étaient aussi. Dix-sept ans plus tard, ces informations mériteraient certainement d'être mises à jour. II serait également pertinent d'examiner les contacts qu'entretiennent les organismes avec les victimes et avec le système judiciaire, deux dimensions qui n'ont pas été abordées dans l'étude.

Comme l'indiquent les résultats, la clientèle des organismes sondés dans la présente étude se compose non seulement de conjoints aux comportements violents, mais aussi d'hommes en difficulté, d'adolescents ou de femmes ayant des comportements violents et, dans une moindre mesure, d'hommes victimes de violence conjugale. Ce phénomène s'explique en partie par le fait que plusieurs programmes québécois ont été mis en place dans les années 1980 par des militants de la condition masculine qui prônaient une approche globale face aux différents problèmes psychosociaux vécus par les hommes (Comité de travail, 2004). II s'explique aussi par des raisons stratégiques. Pour assurer leur développement, les organismes déploient leurs activités dans des champs connexes à l'expertise qu'ils ont développée tant dans le travail auprès des hommes qu'auprès des personnes ayant des comportements violents. Le Québec utilisant relativement peu la voie judiciaire pour la résolution des problèmes sociaux en comparaison avec d'autres régions du Canada et du monde, les organismes doivent diversifier leurs champs d'action pour s'assurer d'un volume d'activité suffisant à leur survie.

Quand on regarde les choses de plus près, on constate que la situation des organismes québécois ne diffère pas radicalement de ce qui se fait ailleurs. Une large proportion de programmes américains et européens traite les femmes aux comportements violents et offre des services aux hommes victimes de violence conjugale (Price et Rosenbaum, 2009 ; WWP, 2008a). Dans le contexte américain, le développement des services destinés aux femmes est lié à la formulation des politiques d'arrestation obligatoires dans certains États. Le développement des pratiques est, là aussi, tributaire de la dynamique sociale dans laquelle s'insèrent les programmes.

Sur le plan organisationnel, quelques similitudes peuvent être observées entre les programmes québécois et américains. D'une part, leur taille semble comparable. On dénombre une médiane de trois employés à temps plein dans les organismes québécois, alors que $85 \%$ 
des programmes américains ont quatre employés à temps plein ou moins (Dalton, 2007). On note, d'autre part, la présence d'une forte proportion d'universitaires au sein du personnel des programmes implantés dans les deux pays. Le recrutement d'intervenants très scolarisés s'explique par la complexité de la problématique et par les enjeux éthiques qu'elle comporte. Compte tenu de l'espoir que la participation d'un conjoint ayant des comportements violents à un programme d'aide suscite chez la victime et des risques qu'elle encourt en cas d'échec de l'intervention, la compétence professionnelle est une préoccupation majeure.

Toujours sur le plan organisationnel, des différences importantes peuvent être observées entre les programmes québécois et leurs homologues sur le plan du financement. Aux ÉtatsUnis, les honoraires des participants constituent souvent la principale, sinon la seule, source de financement des programmes (Dalton, 2007). Plusieurs «n'arrivent pas à bien se développer » dans ce contexte à cause des faibles revenus des participants, des pratiques de tarification graduée selon le revenu et des difficultés à recouvrer les sommes qui leur sont dues (Price et Rosenbaum, 2009: 766). L'accès au financement public, pour ceux qui en reçoivent, tend à devenir plus difficile à cause de l'importance accordée par les décideurs aux interventions basées sur des résultats probants et de la publication d'études ayant questionné l'efficacité des programmes (Gondolf, 2012). En Europe, plusieurs programmes bénéficient de fonds publics, mais leur base de financement n'est pas sécurisée. Les programmes québécois bénéficient à cet égard d'un avantage comparatif grâce à un financement stable que l'État s'est engagé à maintenir dans son dernier plan d'action (Gouvernement du Québec, 2012).

En augmentant le financement public des organismes entre 2003 et 2008, le gouvernement québécois souhaitait mieux répondre aux besoins des conjoints ayant des comportements violents. Cet objectif semble avoir été en partie atteint. Les organismes qui accueillent cette clientèle ont absorbé une hausse des demandes d'aide de $39 \%$ au cours de cette période. Ils ont de plus développé des services complémentaires à l'intervention de groupe, notamment en ayant plus souvent recours à des entrevues de suivi individuel auprès des participants. Bien que le groupe demeure le mode d'intervention privilégié pour permettre aux conjoints ayant des comportements violents de s'entraider, de se confronter et de questionner les valeurs qui soustendent leurs gestes, cette plus grande individualisation du traitement répond à des préoccupations maintes fois exprimées dans la documentation scientifique (p. ex., Cavanaugh et Gelles, 2005). Les organismes ont par ailleurs pu consolider leurs équipes de travail au cours de la période étudiée, ce qui contribue à la qualité des services.

Plusieurs défis demeurent malgré ces avancées. L'un d'eux consiste à maintenir un financement permettant aux organismes de recruter et retenir une main-d'œuvre qualifiée. Les organismes communautaires sont des structures plus légères et moins dispendieuses qui jouent un rôle complémentaire à celui des établissements du réseau de la santé et des services sociaux. Leur action se situe dans le même champ, sans toutefois qu'ils soient en mesure d'offrir des salaires compétitifs aux diplômés universitaires dont ils ont besoin pour rendre leurs services. À titre d'exemple, le salaire horaire moyen des intervenants réguliers des organismes qui ont participé à la présente étude (19,04 \$ en 2007-2008) correspondait à celui versé à un technicien en assistance sociale débutant dans le réseau (19,91 \$ en 2007 ; MSSS, 2009). Le travail en organisme communautaire est souvent vu par les jeunes professionnels comme un 
premier lieu d'insertion professionnelle, voire comme un tremplin vers les établissements offrant de meilleurs salaires. Cela ne favorise pas le maintien de l'expertise au sein des organismes.

Un second défi consiste à répondre de façon appropriée aux besoins financiers des organismes situés en milieu urbain et en milieu rural. Les organismes situés dans les milieux urbains densément peuplés desservent un plus grand bassin de population et ont besoin d'un financement adéquat pour répondre à un grand volume de demandes. En contrepartie, les organismes situés en milieu rural ont souvent de la difficulté à répondre à tous les besoins à partir d'un seul point de service et ont un accès plus restreint à des formations pour leurs employés. Ils doivent donc résoudre des problèmes liés à l'étendue de leur territoire et à leur éloignement. Les résultats de la présente étude suggèrent qu'un financement basé strictement sur une approche populationnelle favorise les organismes situés en milieu urbain et génère des insatisfactions dans les milieux ruraux. Une façon d'équilibrer les besoins des deux types d'organismes pourrait être de pondérer l'indice populationnel qui sert de base au calcul du financement public à l'aide d'autres critères.

\section{CONCLUSION}

Les programmes d'aide aux conjoints ayant des comportements violents ont connu un essor remarquable aux États-Unis au cours des trente dernières années. Depuis, l'expérience a été imitée et adaptée dans de nombreux pays et régions du monde, incluant le Canada. D'autres études sont nécessaires pour voir de quelle façon ces programmes se sont modelés aux réalités nationales et régionales. La présente étude se voulait une contribution à cet effort de recherche. Nous invitons nos collègues de la communauté scientifique à le poursuivre, notamment afin de mieux documenter la réalité canadienne.

BRODEUR, Normand

Professeur

École de service social

Université Laval

LESIEUX, Élisabeth

Cri-Viff

Université de Montréal

RINFRET-RAYNOR, Maryse

École de service social

Université de Montréal

PÉPIN-GAGNÉ, Joannie

Cri-Viff

Université Laval 


\section{BIBLIOGRAPHIE}

Barner, J.R., et M. Mohr Carney (2011). «Interventions for Intimate Partner Violence: A Historical Review », Journal of Family Violence, vol. 26, $n^{0} 3$, p. 235-244. doi:10.1007/s10896-011-9359-3.

Cavanaugh, M.M., et R.J. Gelles (2005). «The Utility of Male Domestic Violence Offender Typologies: New Directions for Research, Policy and Practice », Journal of Interpersonal Violence, vol. 20, no 2, p. 155-166.

Comité de travail en matière de prévention et d'aide aux hommes (2004). Les hommes : s'ouvrir à leurs réalités et répondre à leurs besoins, Québec, ministère de la Santé et des Services sociaux.

Dalton, B. (2007). «What's Going on out There? A Survey of Batterer Intervention Programs », Journal of Aggression, Maltreatment and Trauma, vol. 15, no 1, p. 59-74.

Gondolf, E.W. (2002). Batterer Intervention Systems: Issues, Outcomes, and Recommandations, Thousand Oaks (CA), Sage Publications.

Gondolf, E.W. (2012). The Future of Batterer Programs: Reassessing Evidence-Based Practice, Boston (MA), Northeastern University Press.

Gouvernement du Canada (2008). Répertoire canadien des programmes de traitement pour les hommes violents envers leur conjointe, Ottawa, Travaux publics et Services gouvernementaux Canada.

Gouvernement du Québec (1995). Politique d'intervention en matière de violence conjugale : prévenir, dépister et contrer la violence conjugale, Gouvernement du Québec.

Gouvernement du Québec (2004). Plan d'action gouvernemental 2004-2009 en matière de violence conjugale, Gouvernement du Québec

Gouvernement du Québec (2012). Prévenir, dépister, contrer. Plan d'action gouvernemental 2012-2017 en matière de violence conjugale, Gouvernement du Québec.

Holtzworth-Munroe, A., et J.C. Meehan (2004). « Typologies of Men Who Are Maritally Violent: Scientific and Clinical Implications », Journal of Interpersonal Violence, vol. 19. no 2, p. 13691389.

Mathieu, C., C. Bélanger et $\mathrm{H}$. Brisebois (2006). « Thérapie de groupe pour hommes violents envers leur conjointe: abandon thérapeutique chez ces hommes », Santé mentale au Québec, vol. 31, no 1, p. 169-187.

Ministère de la Santé et des Services Sociaux (MSSS) (1992). Intervention auprès des conjoints violents. Orientations, Québec, ministère de la Santé et des Services sociaux.

Ministère de la Santé et des Services sociaux (MSSS) (2009). Nomenclature des titres d'emploi, des libellés, des taux et des échelles de salaire du réseau de la santé et des services sociaux à partir du 22 novembre 2007. Québec, ministère de la Santé et des Services sociaux. 
Price, B.J., et A. Rosenbaum (2009). «Batterer Intervention Programs: A Report from the Field », Violence and Victims, vol. 24, no 6, p. 757-770.

Rinfret-Raynor, M., N. Brodeur, É. Lesieux et M. Turcotte (2010). Services d'aide en matière de violence conjugale. État de la situation et besoins prioritaires, Montréal, Centre de recherche interdisciplinaire sur la violence familiale et la violence faite aux femmes (Cri-Viff).

Rondeau, G. (1989). Les programmes québécois d'aide aux conjoints violents : rapport sur les seize organismes existants au Québec. Direction générale de la planification et de l'évaluation, ministère de la Santé et des Services sociaux.

Rondeau, G., J. Lindsay, G. Beaudoin et N. Brodeur (1997). Les dimensions éthiques associées à l'intervention auprès des conjoints violents, Montréal, Centre de recherche interdisciplinaire sur la violence familiale et la violence faite aux femmes.

Rosenbaum, A., et P.A. Leisring (2002). « Group Intervention Programs for Batterers », Journal of Aggression, Maltreatment \& Trauma, vol. 5, no 2, 57-71. doi:10.1300/J146v05n02_05.

Statistique Canada (2011). Enquête sur les maisons d'hébergement. En ligne: [http://www23.statcan.gc.ca:81/imdb/p2SV_f.pl?Function=getSurvey\&SDDS=3328\&lang=fr\&d b=imdb\&adm=8\&dis=2] (consulté le 15 juin 2012).

Work with Perpetrators of Domestic Violence in Europe (WWP) (2008a). Récapitulatif de l'enquête. Version 1.0. En ligne: [http://www.work-with-perpetrators.eu/fr/resume.php] (consulté en mai 2013).

Work with Perpetrators of Domestic Violence in Europe (WWP) (2008b). Éléments importants concernant la documentation et l'évaluation des dispositifs d'intervention en direction des hommes auteurs de violences conjugales. Version 1.2. En ligne: [http://www.work-withperpetrators.eu/documents/documentation/wwp_documentation_fr_vers_1_2.pdf?sprache $=d$ ocumentation\%2Fwwp_documentation_fr_vers_1_2.pdf\&submit=Download]. (consulté en mai 2013). 